\title{
ANALYSIS OF PORTABLE MECHANICAL ENVIRONMENTAL MONITORING SYSTEM UNDER ZIGBEE WIRELESS NETWORK
}

\author{
Lu Zhao ${ }^{1}$ \\ ${ }^{1}$ Anhui Vocational College of Electronics \& Information Technology, Bengbu 233000, China. \\ Email: zhaoluanhui2013@126.com
}

\begin{abstract}
To meet the high-quality requirements of current products and make the transmission system more complex and precise, higher requirements are imposed on the safety of mechanical systems. In this research, a portable mechanical environment monitoring system is designed based on ZigBee wireless network technology. First, the overall scheme of the portable mechanical environment monitoring system is designed, and the components of ZigBee wireless network are introduced. Second, to realize a large amount of data transmission, the swing door trending (SDT) algorithm is optimized, and the SDT algorithm based on auto-regressive and moving average model (ARMA) and kurtosis features is proposed. Third, the simulation performance of the traditional SDT compression algorithm and the SDT algorithm based on ARMA and kurtosis characteristics are analysed. The results show that although the traditional SDT compression algorithm performs well in relative error, absolute error, and data compression ratio (DCR) performance, data filtering will occur in the process of data compression. However, there is no such phenomenon in the data compression process of the SDT algorithm based on ARMA and kurtosis characteristics, so the portable mechanical environment monitoring system based on ZigBee wireless network designed in this research has a good monitoring effect on the mechanical environment. Therefore, the results of this research provide a good theoretical support for mechanical environmental monitoring.
\end{abstract}

Keywords: ZigBee Wireless Network; Portable Equipment; Mechanical Environment; Swing Door Trending Compression Algorithm.

\section{Introduction}

With the advancement of global industrialization, the degree of industrial production has become one of the standards to measure the comprehensive strength of a country and an important foundation for national development in the process of fierce competition in globalization [1].

In addition to the precision and complexity of mechanical equipment, the important yardstick to measure the degree of industrial development is the high performance and high reliability of mechanical products. Nowadays, with the rapid development of electronic information technology, network communication technology, and artificial intelligence technology, the degree of industrialization has also increased by leaps and turns. Therefore, ensuring the healthy use of these advanced technologies in industrial development is the current research focus $[2,3]$.

Under normal circumstances, environmental temperature, mechanical vibration, mechanical deformation, and speed of transmission components are important factors to determine whether a mechanical system can work normally in a mechanical environment [4]. High ambient temperature will lead to thermal deformation of components in mechanical equipment, which will reduce the performance and accuracy of equipment. With the increasing accuracy of machinery and instruments, the relationship between temperature and mechanical equipment increases in direct proportion.

Therefore, it is very important to calculate and analyse the thermal deformation of mechanical parts [5].

In addition to the ambient temperature, the deformation factors that affect the mechanical parts are closely related to the mechanical parts' own bearing capacity, design, and materials. Temperature and deformation data play a positive role in analysing the state of the mechanical system and the possible causes of failure [6].

Mechanical vibration data not only reflect the stable state or fault state of equipment operation, but also reflect the demand of production itself. At present, mechanical vibration is very important for mechanical research due to the demand of many machining technologies. In mechanical devices, the transmission device acts as a link, so it is important to monitor the real-time speed of the transmission component to indicate the running state of the device. Traditional mechanical technology has some disadvantages. First, due to the complexity of the 
internal environment of a mechanical gearbox, it is often impossible to directly observe and measure the internal components under test. If the indirect measurement is chosen externally, the test results are inevitably not accurate enough, the noise has a great impact, and the effective signal is weak, which inevitably leads to the difficulty in extracting features of the diagnostic signal at the later stage. Second, for some small wired detection equipment, even if it can be installed in the internal mechanical environment, the device is not practical for some rotating equipment or rotating parts because it needs to read data or power leads. Third, traditional detection equipment is usually one-to-one detection, and detection of multiple targets is often expensive or needs to be redesigned. Finally, the monitoring interface of traditional test equipment is generally dedicated, which may be large, and the installation and maintenance costs are relatively high [7-9].

In view of the shortcomings of traditional mechanical technology, it is urgent to analyse a new mechanical environment monitoring system.

To sum up, in this research, a portable mechanical environment monitoring system based on ZigBee network communication technology is designed. First, the design scheme of the system, the components and optimization of ZigBee network, and the SDT algorithm based on ARMA and kurtosis features are introduced.
Then, the performance of the traditional SDT compression algorithm, the optimized SDT compression algorithm, and the system are tested and analysed. Finally, the portable mechanical environment detection system based on ZigBee wireless network is tested. It is hoped that this study can provide a good idea for the monitoring of mechanical environment.

\section{Methods \\ 2.1 Design of system scheme}

The topological diagram of the portable mechanical environment monitoring system designed in this research is shown in figure 1.

As can be observed from the figure, there are three types of network nodes in ZigBee network, that is, routing nodes, terminal acquisition nodes, and coordinator nodes. All nodes in the ZigBee network are essentially the same and can be configured through software to play different roles, and different types of roles collect different types of data.

The coordinator node is responsible for collecting communication data with Wi-Fi enabled Android devices. Each node runs the FreakZ stack, that is, the ZigBee stack with the Contiki operating system.

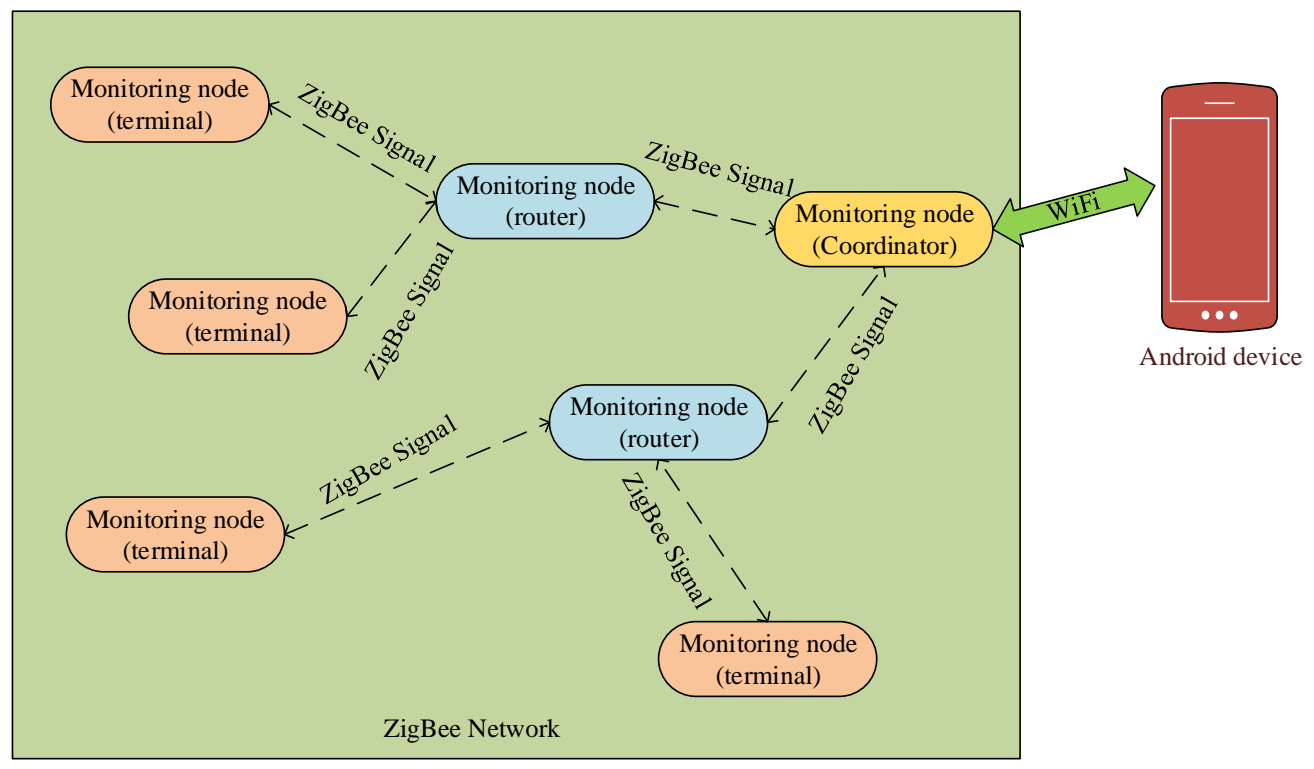

Figure 1: Topology structure of the portable mechanical environmental monitoring system.

The connection between the Android communication device and the ZigBee network is based on the Wi-Fi module. After the connection is established, the Android device sends commands to obtain the required data. The commands flow to the Wi-Fi module and then to the coordinator node in the ZigBee network via a serial port.
Finally, the command is forwarded to the target node. The forwarding process decides whether to use the router node for forwarding according to the distance from the target terminal node. If the distance is far away, the router node should be used for request forwarding. After the request command arrives at the target terminal node, the data 
collection work of each sensor module (including temperature sensing, speed sensing, etc.) begins. After data collection, the data will be transferred to the coordinator node by the same path, and then the data will be transferred to the Android communication device by the WiFi module for the waveform display of the corresponding data.

For data collection under mechanical environment, it needs to have a higher sampling rate and accuracy for data, so there will be an increase in real-time data. Because the traditional sensor network is difficult to realize the wireless transmission of a large amount of original data, in this research, the transmitted data will be compressed by the optimization algorithm. In the transmission process, there is no original data, but the original data will be stored in the hardware node, and the Flash chip will be used for fault analysis.

\subsection{Construction and optimization of ZigBee network}

In this research, each node in the designed system is a Contiki operating system based on FreakZ protocol stack to further realize the construction of ZigBee network. Contiki operating system is a small embedded operating system whose development language is C language. It is an operating system that can support open source and is highly portable, networked, multi-tasking, and non-pre-emptive, and its inter-process communication can be achieved through messages. The Contiki operating system is ideal for a series of memory-constrained network systems, ranging from 8-bit to 32-bit microprocessors. To better match Contiki operating system and FreakZ protocol stack, the system designed in this research will adopt Protothreads model and partial timer [10].

The Protothreads, a thread model for hardwareconstrained threads, is a simplified event driver model. Event-driven model is a popular model in small embedded systems including sensor networks. Compared to a multithreaded system, this system does not need to allocate memory space corresponding to each thread stack, indicating that the event-driven model requires very little memory. For this reason, event-driven models are very suitable for sensor networks, so operating systems such as TinyOS, SOS, and Contiki adopt event-driven models [11]. The event-driven model saves memory overhead, but the programming in this state makes it difficult to write, maintain, and debug state machines.

The new encoding method adopted by Protothreads makes the overhead of each model only two bytes, thus greatly reducing the complexity of the state machine. In addition, it can effectively reduce the number of state machine transfers, thereby reducing the code volume by a third [12].
Contiki operating system sets four timers, namely Timer, CTimer, Etimer, and RTimer. The four timers play different roles in the operation, some provide long time and low-density operation, some provide short time operation, and some provide high density operation or interrupt context.

For FreakZ protocol stack, it belongs to a lightweight ZigBee open source protocol stack, which consists of three parts: four-layer protocols (Media Access Control Layer (MAC Layer), Network Layer (NWK Layer), Application Sub Layer (APS Layer), and Application Framework Layer (AF Layer)), application concepts (ZigBee device objects), and library concepts (ZigBee cluster libraries) [13].

\subsection{SDT process compression algorithm based on ARMA and kurtosis}

SDT algorithm is a compression algorithm that trends straight lines. It can set two pivots on the data points in the vertical direction, and then connect the pivots with the process data to construct two virtual "doors" with pivots as the axis. At the beginning of the algorithm, both doors should still be closed.

After the algorithm starts executing, the two doors open and can't be closed [14]. During the operation of SDT algorithm, all data collected need the same storage space, so the DCR of SDT algorithm is as follows.

$$
\text { Ratio }_{D C R}=\left(1-\frac{\left(N_{s}+1\right) \times 2}{N_{\mathrm{o}}} \times 100 \%\right)
$$

The expression of the data compression absolute error (DCAE) of SDT algorithm is as follows.

$$
\text { Ratio }_{D C A E}=\left(1-\frac{\sum_{i=1}^{N_{\mathrm{o}}}\left|y_{i}-y\right|}{N_{\mathrm{o}}} \times 100 \%\right)
$$

The absolute error of compression is expressed as equation (3).

$$
y_{D C A E}=\frac{x_{D C A E}}{V P P}
$$

Where, $N_{0}$ is the number of original data, $N_{\mathrm{s}}$ is the number of intervals obtained after compression, $y_{\mathrm{i}}$ is the original data, $y$ is the data generated by linear fitting of compressed receipt, and VPP is the peak value of the original data.

Although it can be observed from the above analysis that SDT algorithm is relatively simple and the calculation amount in the process of computing compression is small, there are certain shortcomings in SDT algorithm. If a monitoring point has no way to generate a line at the end of the compression interval that is larger than the position at the end of the recording compression interval due to slow data transformation, the timeliness of online trend extraction can't be guaranteed. 
Since the monitoring system designed in this research has mainly vibration signals, there will be a large number of signals in the actual monitoring, so the actual signal is in an unstable state. Although this shortcoming has no significant influence on the design of this research, in order to ensure the correct operation of the algorithm, the ARMA and kurtosis feature are adopted to improve the SDT compression algorithm [15].

The improved algorithm process can be divided into the following three steps.

Step 1: first, the data is checked to ensure if it is the data stream that was collected for the first time. If it is, the data is transferred to the second step. If not, the data needs to be processed. When $N \times n$ data enter, eigenvalues of $n$ kurtosis $K_{i}(i=1,2, \mathrm{~L}, n)$ need to be calculated. Then the optimal compression threshold ratio $\nabla E B_{i}(i=1,2, \mathrm{~L}, n)$ of each section of data is calculated, and the peak-to-peak values $\nabla P P_{i}(i=1,2, \mathrm{~L}, n)$ of different data are calculated. Finally, the optimal compression threshold of each section of data is calculated according to equation (4).

$$
\nabla E=\frac{E}{\nabla P P}
$$

Where, $E$ is the actual compression threshold, $\nabla P P$ is the peak-to-peak value of the data. SDT algorithm is used to compress the data in real time, and ARMA prediction model is adopted to predict the kurtosis value of the data.

Step 2: if $n+1$ piece of data is coming in, the compression threshold predicted by the previous segment data is used to compress each input data in real time and compare it with the last saved peak-topeak value to refresh the peak-to-peak value $\nabla P P$ at the point that is closest to $m$.

Step 3: if the data is stable (that is, no time is available at the new compression point) for longer than the specified value, the current data is sent as the result of the new compression, and the current data compression is used as the starting point for the new compression.

\section{Results and Discussion}

\subsection{Performance analysis of traditional SDT algorithm}

In this research, to prove the superiority of the proposed SDT compression algorithm based on ARMA and kurtosis characteristics, Mathlab software is used for simulation experiments.

For the performance measurement of traditional SDT algorithm, several groups of different data are adopted to splice. The reason for this selection is that the kurtosis value of the data also keeps changing during the actual monitoring process.
Therefore, in this paper, 150 different kurtosis values are adopted, and the kurtosis values range from 3 to 12, making the data closer to the actual monitored data.

Figure 2 shows the compression effect under different compression threshold ratios.

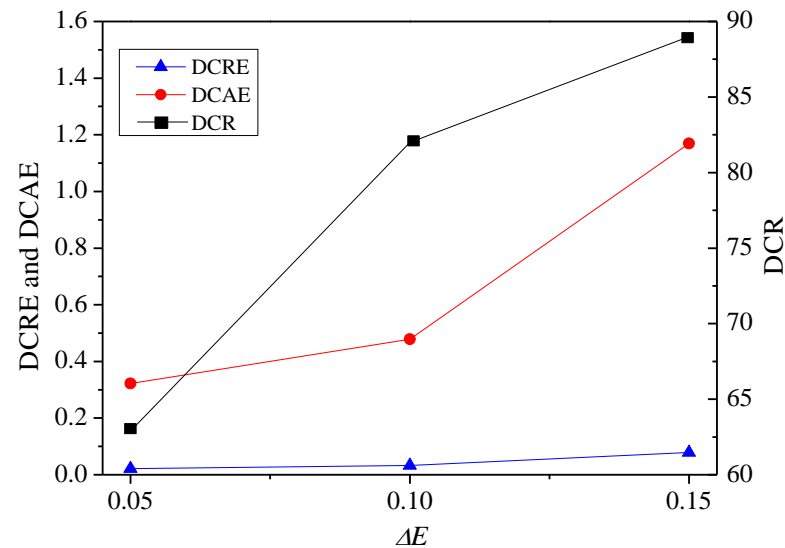

Figure 2: Compression effect diagram under different compression threshold ratios.

As can be observed from the figure, with the increase of the compression threshold ratio, the relative error, absolute error, and DCR increase significantly, and the change trend in figure 2 is exactly the same as that of the traditional SDT algorithm. The relative error of the optimized SDT algorithm is no more than 0.05 , but the maximum compression rate is only $50 \%$. In the three groups of data in the figure, when the compression threshold ratio is 0.1 , the relative error of compression is already greater than that of the optimization algorithm. When the compression threshold ratio is 0.05 , the relative error is very small, and the DCR is still within the acceptable range. It is difficult to observe the defects of the traditional SDT algorithm through the compression effect under different compression thresholds.

When $\Delta E$ is 0.15 , there is no significant difference in compression error. In order to prove the defects of traditional SDT algorithm, the amplitude of compressed data is compared when the compression threshold is 0.15 . As can be observed from figure 3 and figure 4 , the data before compression (figure 3) becomes a straight line after compression by the traditional SDT algorithm (figure 4) after the sequence $0.7 \times 10^{6}$, and a lot of data information is filtered.

According to the principle of SDT algorithm, since the product of compression threshold ratio and peak-to-peak value is too large, the subsequent data is relatively small, so it is regarded as stationary data by the algorithm, and the subsequent data is no longer compressed. The high compression ratio also appears, which obviously shows the shortcomings of the traditional SDT algorithm. 


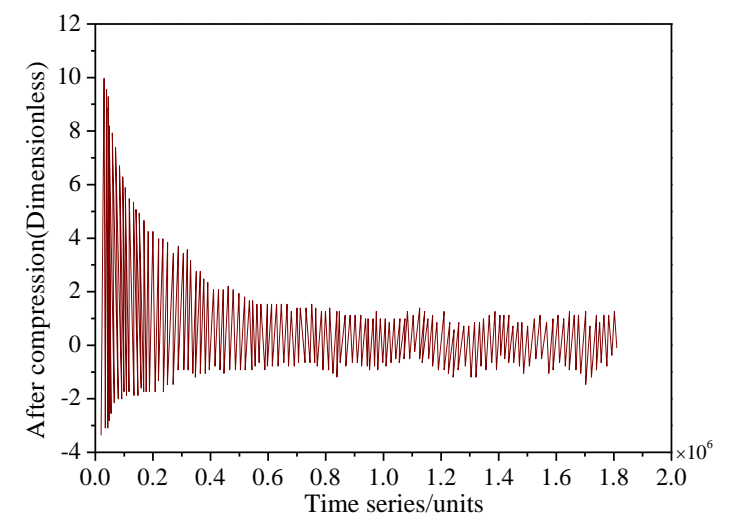

Figure 3: The data amplitude before the overall compression when $\Delta E$ is 0.15 .

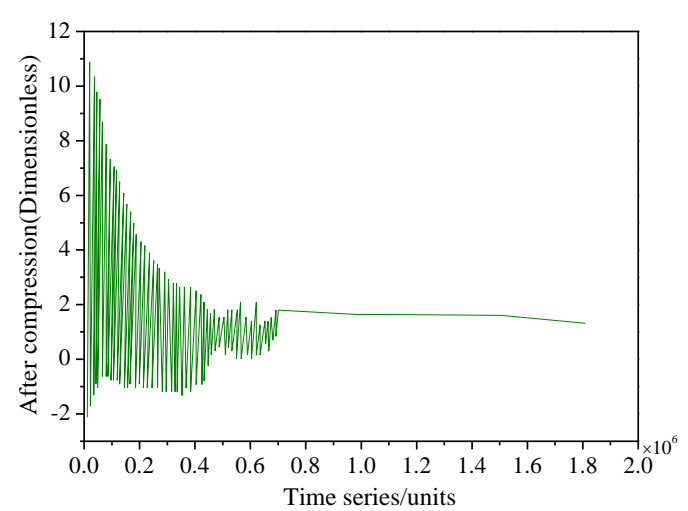

Figure 4: The data amplitude after the overall compression when $\Delta E$ is 0.15 .

\subsection{Performance analysis of SDT algorithm after optimization}

Because the traditional SDT algorithm has some defects, in this research, the optimized algorithm is verified. Consistent with the verification conditions of the traditional algorithm, when is 0.15 , the amplitude of the whole compressed data is compared, as shown in figure 5 and figure 6 . As can be observed from figure 5 and figure 6 , the overall compression effect of the time series on the collected data is much better than that of the traditional SDT algorithm, the distortion in the process of data compression is greatly optimized, and the excellent effect of the improved algorithm is well verified.

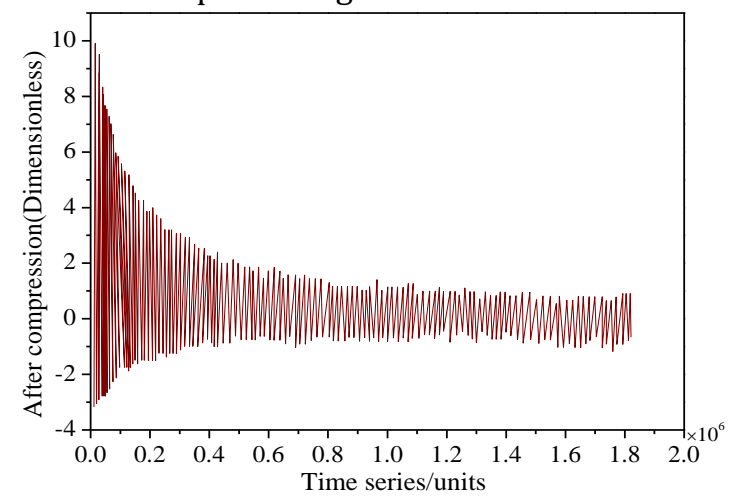

Figure 5: The effect of the optimized SDT algorithm before compression.
Figure 7 is a comparison chart of the tail waveform.

The timing of the tail signal is from $1.7845 \times 106$ to $1.7865 \times 106$. It can be observed from figure 7 that the front and rear compression effects are almost coincident and the compression effect is good.

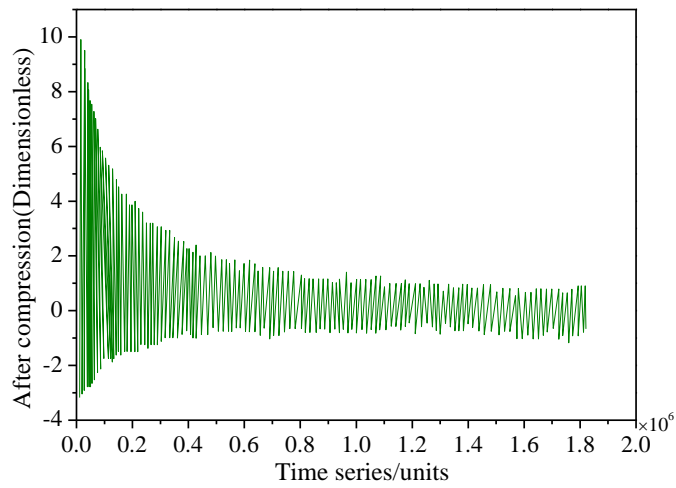

Figure 6: The effect of the optimized SDT algorithm before compression.

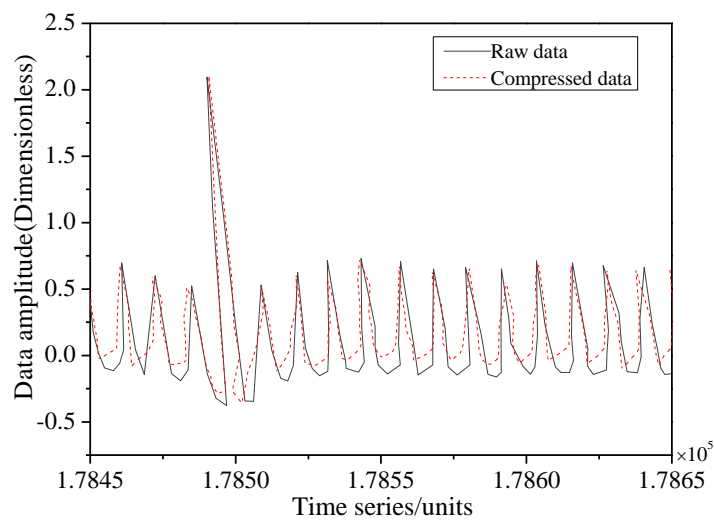

Figure 7: Comparison of the tail waveform.

\subsection{Analysis of the results of system performance tests}

A total of four modules are required for the test of the portable mechanical environment monitoring system, namely, the acquisition terminal, the router, the coordinator, and the android device. However, in this test, data is only collected at the collection terminal node. Mathlab software is used to test the data collected by the system. Although the data types of temperature, acceleration, velocity, and vibration signals are different, they are all voltage signals in essence, so only the compression comparison of vibration signals is conducted in the test.

Figure 8 is a comparison between the original data and the compressed data.

As can be observed from figure 8, the overall change trend of the compressed signal is basically consistent with the original signal, which also indicates that the optimized algorithm proposed in this research can save useful signals under the premise of errors. 
It can be concluded from the compression details that the waveform of the original data is relatively complete in a very short time.

Table 1 shows the comparative analysis results of performance parameters after compression.

As can be observed from table 1 , the system designed in this research controls the relative error of data compression to less than $5 \%$ and compress more than $70 \%$ of the space, thus greatly improving the compression performance.

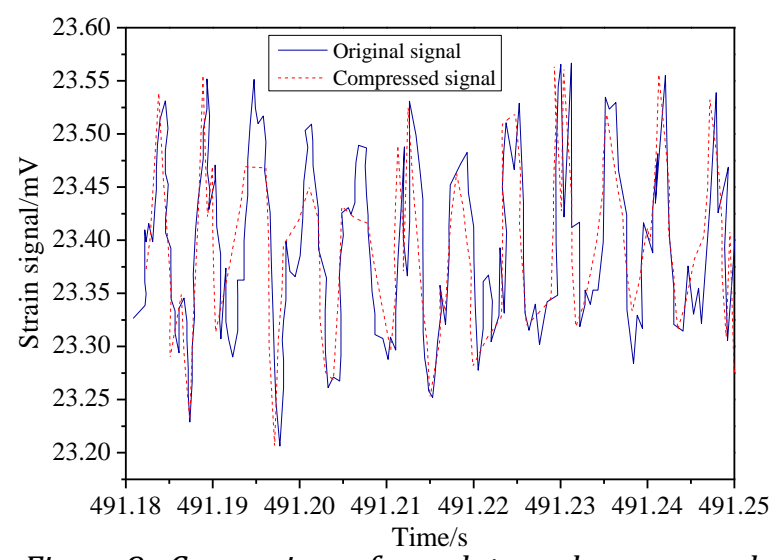

Figure 8: Comparison of raw data and compressed data.

Table 1. comparison of compression parameters.

\begin{tabular}{|c|c|}
\hline Type of compression parameter & Value \\
\hline Signal sampling rate & $1100 \mathrm{~Hz}$ \\
\hline Number of original signal data & 2399800 \\
\hline Number of compressed data & 1513636 \\
\hline DCR & $5.00 \%$ \\
\hline DCRE & 0.3652 \\
\hline
\end{tabular}

\section{Conclusions}

In order to overcome some shortcomings of the traditional mechanical environment monitoring system, a portable mechanical environment monitoring system is designed based on ZigBeee wireless network communication technology. First, the overall scheme of the portable machinery environmental monitoring system is designed, and then the establishment and optimization of ZigBeee wireless network communication technology is introduced. To realize the large amount of data transmission in the portable mechanical environment monitoring system and improve the SDT algorithm, the SDT process compression algorithm based on ARMA and kurtosis features is proposed in this research. Finally, the performance of traditional SDT compression algorithm and the SDT process compression algorithm based on ARMA and kurtosis features is simulated.

The results show that the relative error, absolute error, and DCR of the traditional SDT algorithm increase significantly with the increase of the compression threshold ratio, the relative error is very small, and the DCR is still within the acceptable range. However, in the process of data compression, data loss will occur. The improved SDT algorithm has a better compression ratio, and there is almost no loss in the process of data compression. Therefore, the optimized SDT algorithm has better performance, and its effect of mechanical environment monitoring is better. In this research, the application of wireless communication technology in mechanical environment monitoring system provides a good guidance for mechanical environment monitoring. However, there are still some limitations in this study. In this research, the ZigBee protocol stack is optimized accordingly, but the theoretical transmission speed of ZigBee is $250 \mathrm{kbps}$, while the actual transmission speed would be less than $250 \mathrm{kbps}$ due to some external factors. The theoretical transmission speed of ZigBee designed in this research is $28 \mathrm{kbps}$, and there is still a large space for optimization of ZigBee wireless protocol stack. Subsequent studies can improve the transmission speed of ZigBee to expand the depth of this research.

\section{References}

[1] M. H. Liang, Y. F. He, L. J. Chen, et al., "Greenhouse environment dynamic monitoring system based on WIFI." IFAC-Papers On Line, vol.51, no.17, pp. 736-740, 2018.

[2] B. Li, Y. Ling, M. Tian, et al., "Design and implementationn of sugarcane growth monitoring system based on RFID and ZigBee." International Journal of Online and Biomedical Engineering, vol.14, no.3, pp. 96-106, 2018.

[3] G. A Martín, G. J. Millán, A. Baïri, et al., "Environmental monitoring system based on an open source platform and the Internet of Things for a building energy retrofit." Automation in Construction, vol.87, pp. 201-214, 2018.

[4] Y. Zhang, F. Xu, J.Lei, et al. “Design and research of the smart home system based on CC2530 and ZigBee wireless technology." Journal of Shanghai University of Electric Power, no.2, pp. 16, 2017.

[5] C. T. Yang, S. T. Chen, C. H. Chang, et al., "Implementation of an environmental quality and harmful gases monitoring system in cloud." Journal of Medical and Biological Engineering, vol.39, no.4, pp. 456-469, 2019.

[6] W. Zhang., M. Kumar, J. Yu, et al. "Medical longdistance monitoring system based on internet of things." EURASIP Journal on Wireless Communications and Networking, vol.2018, no.1, pp. 1-8.

[7] J. Fang. "Development of master-slave monitoring systems for automobile exhaust using integration of ZigBee and GSM networks." Photonic Network Communications, vol.37, no.2, pp. 141-152, 2019. 
[8] Q. Zhang, T. Wang, "Design of internet of things method for environmental monitoring based on big data." Ekoloji, vol.28, no.108, pp. 2513-2517, 2019.

[9] K. Priya, G. Shenbagalakshmi, T. Revathi. "Design of smart sensors for real time drinking water quality monitoring and contamination detection in water distributed mains." International Journal of Engineering \& Technology, vol.7, no.1.1, pp. 47-51, 2018.

[10] H. Zhao, W. Yang. "An emergency rescue communication system and environmental monitoring subsystem for underground coal mine based on wireless mesh network." International journal of distributed sensor networks, vol.14, no.10, pp. $1550147718805935,2018$.

[11] D. S. Simbeye. "Industrial air pollution monitoring system based on wireless sensor network." Journal of Information Science and Computing Technologies, vol.6, no.2, pp. 612624, 2017.
[12] S. Li, Z. Huang, F. Rao. "The water quality monitoring system based on GPRS." Mechanical \& Electrical Engineering Technology, no.1, pp. 24, 2017.

[13] D. Xi-bo, W. Jian, L. Bochen, et al. "Design of body temperature and pulse data acquisition system based on Simplici TI wireless network protocol." International Journal of Future Generation Communication and Networking, vol. 10, no. 1, pp. 97-110, 2017.

[14] X. Feng, F. Yan, X. Liu. "Study of wireless communication technologies on Internet of Things for precision agriculture." Wireless Personal Communications, vol.108, no.3, pp. 1785-1802, 2019.

[15] W. Xie, X. Li, X. Long. "Underground operator monitoring platform based on ultra-wide band WSN." International Journal of Online and Biomedical Engineering, vol.14, no.10, pp. 219229, 2018. 